\title{
Research on Modeling of Bionic Porous Scaffold for Bone Defect Repair Based on Bone Mineral Density Distribution
}

\author{
Xueling Bai*, Peng Ding, Peng Zhang and Zhidong Yao \\ Shenzhen Institutes of Advanced Technology, Chinese Academy of Sciences, Shenzhen 518055, China \\ ${ }^{*}$ Corresponding author
}

\begin{abstract}
Porous scaffold is an effective bone substiutes for bone defect repair. The research developed a new method of modeling of bionic porous scaffold for bone defect repair based on bone mineral density distribution through topological analysis and mathematical modeling of porous scaffold, and, taking femur segmental defect as sample, validated the feasibility and accuracy of this method of modeling of bionic porous scaffold for bone defect repair based on bone mineral density distribution. The method provides a powerful tool for 3D printing precision modeling in bone defect repair, superior to others traditional uniform porous scaffold, a bionic porous scaffold based on bone mineral density distribution undoubtedly provide a better microstructure and microenvironment which has bionic biomechanical characteristics of bone defect and appropriate bionic micropore size and porosity for cell migration, growth, proliferation, differentiation etc.
\end{abstract}

Keywords—bionic; porous scaffold; bone defect; bone Mineral Density; 3D printing

\section{INTRODUCTION}

Bone defect, which is possibly caused by trauma, tumor, infection and congenital bone diseases etc., is a clinical common disease, and it is also one of the difficult problems in clinical treatments. In order to overcome the limitations of traditional autogenous and allogenous bone grafts, it is the main object for many scholars to search for effective live transplantation artificial bone materials as bone substiutes [1]. 3D printing porous scaffolds is an effective approach for bone defect repair, which provides the microstructure and microenvironment for cell migration, growth, proliferation and differentiation, the mechanical stimulation on the expression of osteogenesis, and the osteoinductive properties of 3D printing biomaterials [2-4].

However, the porosity and micropore size of porous scaffold 3D printed by traditional 3D modeling methods using Mimics, Simpleware, Amira etc., is usually uniform and regular, only can guarantee porosity, micropore size, biomechanical characteristics and physiological microenvironment of a few bone defect region, there is still a big gap in comparison with the real anatomic microstructure and physiological function of bone tissue, which possibly result in the dead cavity formation during tissue regeneration and become a new therapeutic risk $[5,6]$. The ideal porous scaffold for bone defect repair need meet: 1) personalized external shape suiting for irregular bone defect shape; 2) patient-specfic bionic porous scaffold meeting the biomechanical characteristics of bone defect; 3) appropriate bionic micropore size and porosity of porous scaffold meeting the microstructure and the microenvironment of bone defect for cell migration, growth, proliferation, differentiation etc.

This research developed a new method of modeling of bionic porous scaffold for bone defect repair based on bone mineral density (BMD) distribution, through topological analysis and mathematical modeling of porous scaffold microstructure, the bionic microstructure constructed by this method can provide bionic porosity and micropore size which can be adjusted by BMD distribution, and provide approximate biomechanical characteristics and microenvironment similar to real bone defect region. The method provides a powerful tool for 3D printing precision modeling in bone defect repair.

\section{MATERIALS AND METHODS}

\section{A. Topological Analysis of Bionic Porous Scaffold}

A porous scaffold is composed of a lot of microstructural units with the same topological microstructure, which are orderly arranged in $\mathrm{X}, \mathrm{Y}$ and $\mathrm{Z}$ directions. This research takes a kind of porous scaffold in a specific topological microstructure as sample to explain how to realize modeling of bionic porous scaffold for bone defect repair based on BMD distribution, other kinds of bionic porous scaffolds in different topological microstructures can be modeled in the same method.

In this porous scaffold model (see Figure 1A), the structure of any microstructural unit shown in Figure 1B, has 32 vertices and 30 facets with same topological microstructure and square section of trabecula. Assuming the central point of microstructural unit as the basepoint for locating the position of microstructural unit in porous scaffold, all 32 coordinates of any vertices can be calculated based on their relative spatial relation with the central point. Thus, according to the STL construction rules "Standard Triangle Language" and "Standard Tessellation Language", the normal and relevant vertices (ordered by the right-hand rule) of triangle patches of any microstructural unit can be designed in a three-dimensional Cartesian coordinate system. All triangle patches of all microstructural units will be merged together into a STL model of porous scaffold. 


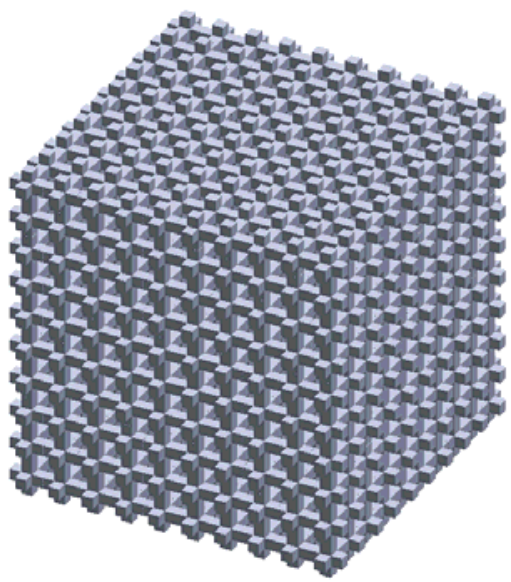

A)

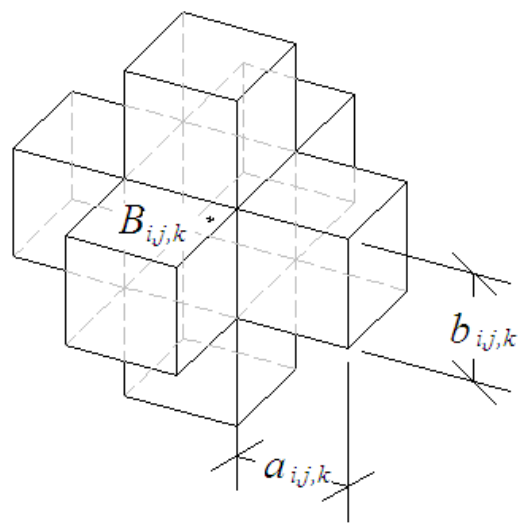

B)

FIGURE I. POROUS SCAFFOLD. A) $10 \times 10 \times 10$ POROUS SCAFFOLD, B) MICROSTRUCTURAL UNIT.

In this porous scaffold model, the microstructural units are used to simulate trabecular bone, when all microstructural units are in the same size, the porous scaffold presents uniform porosity and micropore size, while every microstructural unit can be adjusted by BMD collected from relevant position in bone defect region, porous scaffold will present bionic porosity and micropore size closely related to BMD distribution, additional, when the value of BMD of some microstructural units, is equal to zero or smaller than a predetermined value, these microstructural units was ignored, thus, irregular porosity and different micropore size can be simulated approximately similar to the real bone.

\section{B. Mathematical Modeling of Bionic Porous}

\section{1) Uniform porous scaffold model}

It is assumed, the apparent size length, width and height of porous scaffold, can be achieved from external cuboid of bone defect, the microstructural units of this porous scaffold are arranged in $\mathrm{m}$ rows, $\mathrm{n}$ columns and s layers, which are equal to the ratio of length, width, height with the size of microstructural unit, respectively. The external size of microstructural unit is decided by the micropore size (length of the trabecular bone of microstructural unit) and the thickness of the trabecular bone of microstructural unit, thereinto, the micropore size and the thickness of the trabecular bone of microstructural unit can be determined by engineer's experiences or experimental measurements. Average BMD is set equal to average value of BMD of all pixels within bone defect region which can be calculated form CT values.

$$
\begin{gathered}
\mathrm{m}=\mathrm{L} / \mathrm{L}_{0} \\
\mathrm{n}=\mathrm{W} / \mathrm{L}_{0} \\
\mathrm{~s}=\mathrm{H} / \mathrm{L}_{0} \\
\mathrm{~L}_{0}=2 \mathrm{a}_{0}+\mathrm{b}_{0} \\
\mathrm{~V}_{0}=\mathrm{L}_{0}^{3} \\
\mathrm{~V}=6 \mathrm{a}_{0} \mathrm{~b}_{0}{ }^{2}+\mathrm{b}_{0}{ }^{3} \\
\mathrm{P}_{0}=\left(\mathrm{V}_{0}-\mathrm{V}\right) / \mathrm{V}_{0}=\left(8 \mathrm{a}_{0}{ }^{3}+12 \mathrm{a}_{0}{ }^{2} \mathrm{~b}_{0}\right) /\left(2 \mathrm{a}_{0}+\mathrm{b}_{0}\right)^{3} .
\end{gathered}
$$

where, L, W, and $\mathrm{H}$ present the length, width, height of porous scaffold achieved from apparent shape of bone defect, respectively, $\mathrm{L}_{0}$ presents the external size of the microstructural unit, $m, n, s$ present the number of row, column and layer of the microstructural unit in porous scaffold, respectively, $\mathrm{a}_{0}$ and $b_{0}$ present half-length and thickness of the trabecular bone of microstructural unit of uniform porous scaffold, respectively, $\mathrm{P}_{0}$ presents average porosity of porous scaffold, $\mathrm{V}_{0}$ presents apparent volume of the microstructural unit, $\mathrm{V}$ presents real volume of the microstructural unit.

Assumed the basepoint coordinate of first microstructural unit in porous scaffold is set to equal to an appointed point or zero point, while, the basepoint coordinate of any microstructural unit can be achieved by arithmetic progression, then, the coordinates of all 32 vertices of any microstructural unit can be calculated based on their relative spatial relation with the central point. Finally, after triangle patches of all microstructural unit being constructed, remarkablely, 50 triangle patches in 25 facets of any frontier microstructural unit are constructed excluding 5 end facets which are repeated with 5 adjacent microstructural units, 48 triangle patches in 24 facets of any of other microstructural units are constructed excluding 6 end facets which are repeated with 6 adjacent microstructural units, all triangle patches of all microstructural units are merged together into a STL model of porous scaffold.

$$
\begin{aligned}
& B_{i, j, k}(x, y, z)=B_{0,0,0}(x, y, z)+\left[j L_{0}, i L_{0}, k L_{0}\right] . \\
& V^{n_{i, j}, k}(x, y, z)=F_{n}\left(B_{i, j, k}(x, y, z), a_{i, j, k}, b_{i, j, k}\right) .
\end{aligned}
$$


where, $a_{i, j, k}$ and $b_{i, j, k}$ present half-length and thickness of the trabecular bone of the microstructural unit in the i-th row, j-th column and k-th layer, respectively, $\mathrm{B}_{0,0,0}(\mathrm{x}, \mathrm{y}, \mathrm{z})$ presents the basepoint coordinate of first microstructural unit (lower-left corner) of porous scaffold, $\mathrm{B}_{\mathrm{i}, \mathrm{j}, \mathrm{k}}(\mathrm{x}, \mathrm{y}, \mathrm{z})$ presents the basepoint coordinate of the microstructural unit of porous scaffold in the $\mathrm{i}$-th row, j-th column and k-th layer, $\mathrm{n}$ presents the serial number of the vertex of any microstructural unit of porous scaffold, $\mathrm{V}_{\mathrm{i}, \mathrm{j}, \mathrm{k}}(\mathrm{x}, \mathrm{y}, \mathrm{z})$ presents the coordinate of the $\mathrm{n}$-th vertex of any microstructural unit in porous scaffold.

\section{2) Bionic porous scaffold model}

Based on the uniform porous scaffold model, the micropore size can be adjusted by BMD, the new thickness of the trabecular bone of microstructural units can be calculated on the external size of the microstructural unit, thus, the coordinates of all 32 vertices of any microstructural unit can be obtained. The porosity of microstructural unit and of whole porous scaffold after being adjusted can also be achieved.

$$
\begin{gathered}
a_{i, j, k}=c_{0} D(i, j, k) / D_{0} . \\
b_{i, j, k}=L_{0}-2 a_{i, j, k} .
\end{gathered}
$$

$$
\begin{gathered}
P(i, j, k)=\left(8 a_{i, j, k}{ }^{3}+12 a_{i, j, k}{ }^{2} b_{i, j, k}\right) /\left(2 a_{i, j, k}+b_{i, j, k}\right)^{3} \\
P_{a}=\sum_{k=0}^{s} \sum_{j=0}^{n} \sum_{i=0}^{m_{i=0}} P(i, j, k) / m n s
\end{gathered}
$$

where, $\mathrm{D}_{0}$ presents initial apparent bone density, c presents the weighted coefficient, $D(i, j, k)$ and $P(i, j, k)$ present BMD and porosity of microstructural unit in the $\mathrm{i}$-th row, $\mathrm{j}$-th column and $\mathrm{k}$-th layer, respectively, $\mathrm{P}_{\mathrm{a}}$ presents average porosity of whole porous scaffold.

After the coordinates of all 32 vertices of any microstructural unit being adjusted based on new micropore size and thickness, all triangle patches of microstructural units can be constructed through designing unit normal and relevant vertices, thereinto, when the value of BMD of some microstructural unit, is equal to zero or smaller than a predetermined value, these microstructural units were ignored. All triangle patches of microstructural units are merged together into a STL model of bionic porous scaffold for bone defect repair based on BMD distribution.

\section{Algorithm Flow for Bionic Porous Scaffold Modeling}

This research, taking femur segmental defect as sample, construct bionic porous scaffold model. The algorithm flow for bionic porous scaffold modeling is as follows,

1) Segmentation: Segment the individual femur segmental defect, the defect information in bone defect region can be collected by the contralateral normal side, construct the geometric model of external cuboid of femur segmental defect, and determine the length $\mathrm{L}$, width $\mathrm{W}$, height $\mathrm{H}$ of porous scaffold achieved from external size of bone defect.
2) BMD calculatation: Collect the CT value of all pixels of all pixels in all level layers, calculate BMD of all pixels, and calculate the average $\mathrm{BMD}_{0}$ of bone defect.

3) Parameters determination of uniform porous scaffold: Determine the half-length $a_{0}$ and thickness $b_{0}$ of the trabecular bone of microstructural unit of uniform porous scaffold, calculate the apparent size of the microstructural unit $\mathrm{L}_{0}$ by (4) and the number of row $\mathrm{m}$, column $\mathrm{n}$, layer $\mathrm{s}$ by (1-3), and calculate the porosity $\mathrm{P}_{\mathrm{a}}$ of uniform porous scaffold by (5-7).

4) $B M D$ value determination of microstructural units: Query the BMD value of all microstructural units of first layer with an interval of microstructure size $\mathrm{L}_{0}$ in $\mathrm{X}$, $\mathrm{Y}$ directions, and calculate the bone density $\mathrm{D}(\mathrm{i}, \mathrm{j}, \mathrm{k})$ of all microstructural units of others layers by linear interpolation.

5) Coordinates calculatation of porous scaffold: Appoint the basepoint $\mathrm{B}_{0,0}, 0(\mathrm{x}, \mathrm{y}, \mathrm{z})$ of first (lower-left corner) microstructural unit of porous scaffold, calculate the basepoint coordinate $B_{i, j, k}(x, y, z)$ of any microstructural unit of porous scaffold in the i-th row, j-th column and k-th layer by (8), and calculate the coordinate $\mathrm{V}_{\mathrm{i}, \mathrm{j}, \mathrm{k}}(\mathrm{x}, \mathrm{y}, \mathrm{z})$ of the $\mathrm{n}$-th vertex of any microstructural unit in porous scaffold by (9).

6) STL modeling of uniform porous scaffold: Construct all triangle patches of microstructural units, and merge them together into a STL model of uniform porous scaffold.

7) Adjusting microstructural units by BMD: Adjust the microstructure sizes by bone density distribution by (10-11), and calculate the coordinates of 32 vertices of any microstructural units of bionic porous scaffold by (9), thereinto, when the value of BMD D(i, j, k) of some microstructural unit, is equal to zero or smaller than a predetermined value, the triangle patches of these microstructural units were ignored, then, calculate the porosity $\mathrm{P}_{\mathrm{a}}$ of bionic porous scaffold by (12-13).

8) STL modeling of bionic porous scaffold: Construct all triangle patches of microstructural units regulated by bone density distribution, and merge them together into a STL model of bionic porous scaffold.

\section{RESUlTS}

Figure 2 shows the geometric model of femur segmental defect by Mimics based on 39 DICOM files, the defect information in bone defect region can be collected by the contralateral normal side. The uniform porous scaffold STL model (see Figure 3) of external cuboids of femur segmental defect was constructed with the same topological microstructure as Figure 1B. After adjusting the microstructure sizes by bone density distribution, calculating the coordinates of 32 vertices of any microstructural units of bionic porous scaffold, and constructing triangle patches of all microstructural units, the bionic porous scaffold model for femur segmental defect repair based on bone density distribution was constructed, shown in Figure 4, which presents the same appearance morphology with the geometric model of femur segmental defect (Figure 2) and bionic microstructure based on BMD distribution, because the value of BMD of all microstructural units locating at the exterior of femur and 
medullary cavity, is equal to zero, these microstructural units were ignored .

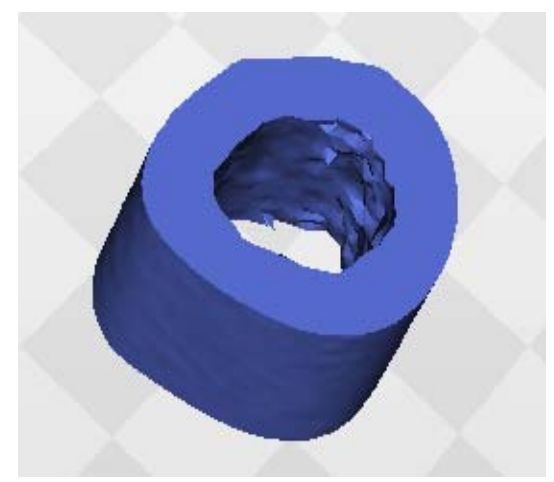

FIGURE II. THE GEOMETRIC MODEL OF FEMUR SEGMENTAL DEFECT BY MIMICS.

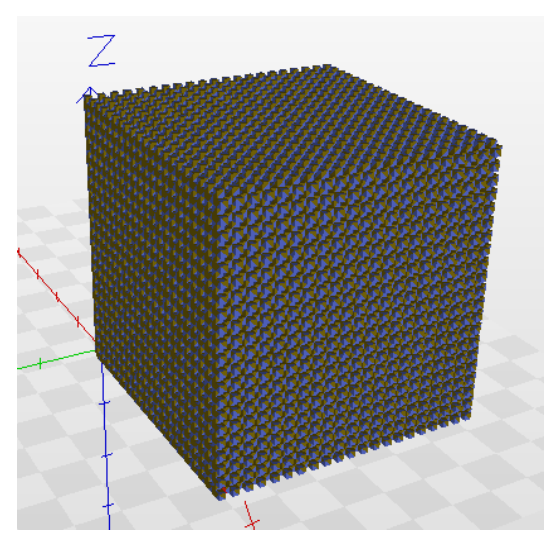

FIGURE III. THE UNIFORM POROUS SCAFFOLD STL MODEL OF EXTERNAL CUBOIDS OF FEMUR SEGMENTAL DEFECT.

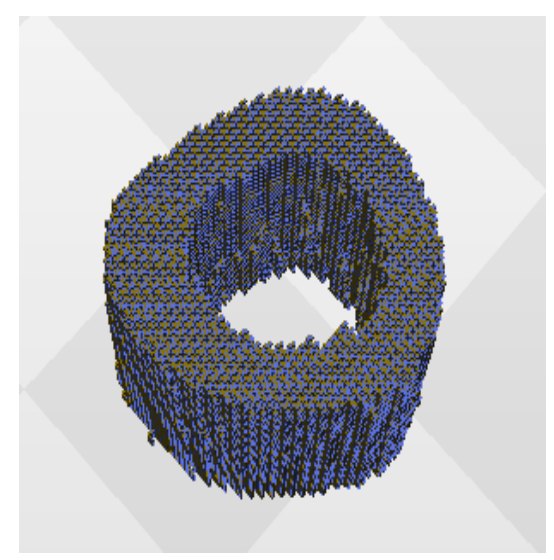

FIGURE IV. BIONIC POROUS SCAFFOLD STL MODEL FOR FEMUR SEGMENTAL DEFECT REPAIR BASED ON BONE DENSITY DISTRIBUTION.

\section{CONCLUSION AND Discussion}

3D printing porous scaffolds as bone substiutes is an effective approach for bone defect repair. Superior to others traditional uniform porous scaffold, a bionic porous scaffold based on BMD distribution for bone defect repair can undoubtedly provide a better microstructure and microenvironment, which have bionic biomechanical characteristics of bone defect and appropriate bionic micropore size and porosity for cell migration, growth, proliferation, differentiation etc.

The research developed a new method of modeling of bionic porous scaffold for bone defect repair based on BMD distribution. At the same time, the research, taking femur segmental defect as sample, construct successfully bionic porous scaffold STL model (Figure 4) for femur segmental defect repair based on bone density distribution which has the same appearance morphology with the geometric model of femur segmental defect (Figure 2) and bionic microstructure based on BMD distribution. The modeling method of bionic porous scaffold for bone defect repair based on BMD distribution provides a powerful tool for 3D printing precision modeling in bone defect repair.

In this research, only a kind of porous scaffold in a specific topological microstructure (Figure 1B) was topologically analyzed and mathematically modeled to realize modeling of bionic porous scaffold for bone defect repair based on BMD distribution, other kinds of porous scaffolds in a different topological microstructure can be modeled based on BMD distribution by the same method. Besides, in the modeling of bionic porous scaffold for femur segmental defect repair based on bone density distribution, the topological kind of microstructure, the initial parameters including the micropore size, the thickness of the trabecular bone and the weighted coefficient $c$ determined by author only validate the feasibility and accuracy of the method of modeling of bionic porous scaffold for bone defect repair based on BMD distribution, which can be optimized and validated through animal controlled experiments based on different sclerotin conditions, bone defect anatomic regions and cancellous bone defects or cortical bone defects, and the physical and chemical properties of $3 \mathrm{D}$ printing materials.

\section{ACKNOWLEDGMENT}

This work was supported by the Shenzhen science and technology project with the Grant No. JCYJ20150401150223635 and JCYJ20170818164059405.

\section{REFERENCES}

[1] A.J. Salgado, O.P. Coutinho, R.L. Reis, "Bone tissue engineering: state of the art and future trends.” Macromol Biosci. 2004, 4(8): 743-765.

[2] L.H. Nguyen, N. Annabi, M. Nikkhah, H. Bae, L. Binan, S. Park, Y. Kang, Y. Yang, A. Khademhosseini, "Vascularized Bone Tissue Engineering: Approaches for Potential Improvement,” Tissue Eng Part B Rev. 2012, 18(5): 363-382.

[3] J.A. Inzana, D. Olvera, S.M. Fuller, J.P. Kelly, O.A. Graeve, E.M. Schwarz, S.L. Kates, H.A. Awad, "3D printing of composite calcium phosphate and collagen scaffolds for bone regeneration,” Biomaterials, 2014, 35(13): 4026-4034.

[4] A.M. Wojtowicz, A. Shekaran, M.E. Oest, K.M. Dupont, K.L. Templeman, D.W. Hutmacher, R.E. Guldberg, A.J. García, “Coating of biomaterial scaffolds with the collagen-mimetic peptide GFOGER for bone defect repair,” Biomaterials, 2010, 31(9): 2574-2582.

[5] K.F. Leong, C.M. Cheah, C.K. Chua, "Solid freeform fabrication of three-dimensional scaffolds for engineering replacement tissues and organs,” Biomaterials, 2003, 24 (13): 2363-2378.

[6] T.A. Desai, "Micro- and nanoscale structures for tissue engineering constructs,” Med Eng Phys. 2000, 22(9): 595-606. 